\title{
Renal pathology and clinical associations in systemic sclerosis: a historical cohort study
}

This article was published in the following Dove Press journal:

International Journal of General Medicine

\author{
Pantipa Tonsawan' \\ Komkid Talabthong' \\ Anucha Puapairoj ${ }^{2}$ \\ Chingching Foocharoen' \\ 'Department of Medicine, Faculty of \\ Medicine, Khon Kaen University, Khon \\ Kaen, Thailand; ${ }^{2}$ Department of \\ Pathology, Faculty of Medicine, Khon \\ Kaen University, Khon Kaen, Thailand
}

Background: The information guiding the treatment decision(s) for renal diseases in systemic sclerosis (SSc) is the renal pathological finding. This study aimed to evaluate the renal pathological diagnosis and its clinical feature among SSc.

Method: A historical cohort study was performed on adult Thai SSc patients who underwent renal biopsy during January 2005-December 2016. The renal pathologic findings and patient clinical characteristics were reviewed. Chi-square or Fisher's exact test was applied to analyze the association between clinical manifestation and renal pathology.

Results: Of the 26 SSc patients identified (77\% female), $46 \%$ had the diffuse cutaneous SSc subtype. The mean age at the time of biopsy was $53.2 \pm 14.4$ years and median duration of disease was 2.4 years (IQR 0.5-7.0). Rapidly progressive glomerulonephritis (RPGN) was the most common renal manifestation (53.9\%) followed by nephrotic syndrome $(19.2 \%)$ and nephritis (11.5\%). The pathological diagnosis included lupus nephritis (LN) class IV (26.9\%), LN class V (19.2\%), scleroderma renal crisis (SRC; 19.3\%), progressive renal disease in scleroderma (7.7\%), and IgA nephropathy (7.7\%). The nephrotic syndrome was the most common renal feature among LN class $\mathrm{V}$ patients, whereas RPGN was the commonest renal presentation among LN class IV and SRC patients $(p=0.001)$. Dialysis treatment at the time of kidney biopsy was significantly higher in SRC patients than in the other groups $(p<0.001)$. The SRC tended to have more frequent cardiac involvement, pulmonary fibrosis, and shorter disease duration than the other groups.

Conclusion: This is the first report of renal pathologic findings in Thai SSc patients. RPGN is the commonest renal manifestation among SSc who underwent kidney biopsy; for whom LN was the most common pathological finding. Nephrotic syndrome is a clinical feature of glomerular diseases other than renal involvement in SSc.

Keywords: scleroderma, renal biopsy, glomerular disease, scleroderma renal crisis

\section{Introduction}

Systemic sclerosis (SSc) is a systemic connective tissue disease characterized by progressive skin fibrosis. SSc is classified into two types distinguished by the extent of skin involvement: limited cutaneous systemic sclerosis (lcSSc) and diffuse cutaneous systemic sclerosis (dcSSc). ${ }^{1,2}$ Renal complications are common which the prevalence was reported to be around $50 \%$, and the prevalence was up to $60-$ $80 \%$ per autopsy. ${ }^{3-5}$ Moreover, in some diseases it may contribute to end-stage kidney disease and mortality. ${ }^{6,7}$ The renal manifestation varies depending on the characteristics of renal abnormalities, ${ }^{4,8}$ which the spectrum of the disease include scleroderma renal crisis (SRC), normotensive renal crisis, anti-neutrophil cytoplasmic antibodies (ANCA)-associated glomerulonephritis, penicillamine-associated
Correspondence: Chingching Foocharoen Department of Medicine, Faculty of Medicine, Khon Kaen University, Khon Kaen, 40002 Thailand

Tel +6643363746

Email fching@kku.ac.th 
renal disease, proteinuria, microalbuminuria, and isolated reduction in glomerular filtration rate (GFR). ${ }^{8}$

$\mathrm{SRC}$ is a severe renal complication in SSc, which typically occurs in the first 4 years after disease onset, and more so among those diagnosed with dcSSc subset. ${ }^{2,6}$ The prevalence of SRC varies: $2-15 \%$ in $\mathrm{dcSSc}$ and $0.5-$ $0.6 \%$ in lcSSc. ${ }^{9,10}$ The classic presentation of SRC is acute kidney injury (AKI), new onset of anemia, and rapid onset of accelerated hypertension. ${ }^{11,12}$ Proteinuria can be detected in SRC, but it is less likely to be greater than $2 \mathrm{~g} / \mathrm{d}$ or in a nephrotic range. ${ }^{13,14}$

Kidney biopsy is an important investigation that can identify the nature of the disease especially the treatable renal condition in SSc patient; such as inflammatory glomerular disease (LN and renal involvement in systemic vasculitis), which commonly presents with an unexplained decline in GFR, proteinuria, and microscopic hematuria. ${ }^{15}$ Kidney biopsy is thus helpful for making a definite diagnosis and for guiding treatment planning.

In Thailand, the prevalence of renal involvement in dSSc was approximately $4 \%$ and SSc overlap with other connective tissue disease was $17 \%$ of which SSc-systemic lupus erythematosus (SLE) overlap syndrome was the second most common type (16\%). ${ }^{16,17}$ Renal involvement in SSc and SSc-SLE overlap syndrome has been reported in Thai SSc. ${ }^{17,18}$ However, the renal pathologic finding in SSc is limited. This study thus aimed to evaluate a renal pathological diagnosis and its clinical association among Thai SSc patients.

\section{Materials and methods}

We conducted a historical cohort study of SSc patients over 18 years who had undergone kidney biopsy and followed up at Srinagarind Hospital, Khon Kaen University, Khon Kaen, Thailand, between January 2005 and December 2016. The kidney biopsy was determined by a nephrologist and the uniform indications include nephrotic syndrome, persistent proteinuria more than $500-1000 \mathrm{mg} /$ day with or without hematuria, unexplained AKI, or chronic kidney disease. ${ }^{19}$ All renal biopsies were processed using standard techniques for light microscope and immunofluorescence and were then interpreted by renal pathologist.

The medical charts were reviewed and included details on age, age of SSc diagnosis, sex, SSc subset, date of first detected SSc symptom, modified Rodnan skin score (mRSS), internal organ involvement, baseline comorbidities, systolic blood pressure (SBP), diastolic blood pressure (DBP), blood urea nitrogen (BUN), serum creatinine, estimated glomerular filtration rate (eGFR) by using Chronic Kidney Disease Epidemiologic (CKDEPI) equation, $24 \mathrm{hrs}$ protein excretion or urine protein-creatinine ratio, urinalysis, and medical treatment.

\section{Operation definition}

The diagnosis of scleroderma was based on the 1980 American College of Rheumatology (ACR) criteria $^{20}$ and/or 2013 ACR/European League Against Rheumatism (EULAR) classification. ${ }^{21}$ Scleroderma was classified as either $1 \mathrm{cSSc}$ or dcSSc subset as per the classification created by LeRoy et al. ${ }^{22}$ The definition of SSc overlap syndrome was met when the patient fulfilled the criteria for scleroderma and they had the clinical features or specific autoantibodies of a connective tissue disease. ${ }^{17,23,24}$

Pulmonary fibrosis was confirmed when interstitial fibrosis was detected by either chest radiograph or highresolution computed tomography (HRCT) of the chest, most pronounced in the basilar portions of the lungs, or presence of Velcro crackles on auscultation not due to another cause. ${ }^{21}$ Cardiac involvement was defined by the detection of pericardial effusion, ejection fraction $<50 \%$, or unexplained cardiac arrhythmia. Pulmonary arterial hypertension (PAH) was defined by a mean pulmonary arterial pressure $\geq 25 \mathrm{mmHg}$ and a pulmonary capillary wedge pressure $<15 \mathrm{mmHg}$ from right heart catheterization. ${ }^{21,25}$

Esophageal involvement was defined as the presentation with dysphagia, heartburn, and regurgitation. ${ }^{26,27}$ Intestinal involvement was defined as the clinical manifestation with diarrhea, constipation, malabsorption, chronic intestinal pseudo-obstruction, and other causes were excluded. $^{27}$

AKI was defined as an increase of serum creatinine $\geq 0.3 \mathrm{mg} / \mathrm{dL}$ from baseline or increase of serum creatinine $>1.5$ times baseline that is known or presumed to have developed within 7 days serum creatinine $>1.2 \mathrm{mg} / \mathrm{dL}$ at initial presentation. ${ }^{4,28}$

Nephritis was defined as red blood cell (RBC) in centrifuged urine $>5$ cells/high power field (HPF), detected dysmorphic RBC, or RBC cast with proteinuria. Nephrotic was defined as the proteinuria $>3000 \mathrm{mg} / 24 \mathrm{hrs}$ or spot urine protein-creatinine ratio $>3000 \mathrm{mg}$ of protein/gm of creatinine. $^{29}$

Rapidly progressive glomerulonephritis (RPGN) was defined as the presentation with clinical syndrome characterized by nephritis with a rapid decline in GFR from a week to several months. ${ }^{29}$ 
LN was defined by the renal pathologist according to the International Society of Nephrology/Renal Pathology Society (ISN/RPS) 2003 classification. ${ }^{30}$

Briefly, the classes were defined as follows: class I - minimal mesangial LN, class II - mesangial proliferative LN, class III - focal LN, class IV - diffuse LN, class $\mathrm{V}$ - membranous LN, and class VI - advanced stage LN.

SRC was defined as a renal pathology demonstrated by obliterative vasculopathy with an onion skin appearance of the arterioles or interlobular arteries in the chronic stage, and/or mucoid changes of the arterioles or interlobular arteries in the subacute stage, and/or fibrinoid necrosis of the arterioles or interlobular arteries in the acute stage, assuming other causes were excluded. ${ }^{31}$

Progressive renal disease in scleroderma was defined by the non-specific abnormalities within arteries, glomerulus, and interstitium that cannot be explained by SRC or other etiologies. ${ }^{15,32}$

\section{Statistical analysis}

The clinical characteristics were categorized as being dichotomous, polytomous, or continuous variables. The categorical data were tested for significance using the $\chi^{2}$ or Fisher exact test (as appropriate). The respective student $t$-test and analysis of variance (ANOVA) were used to test for differences between means of the dichotomous and polytomous categorical data. The Wilcoxon Ranksum or Kruskal-Wallis test was applied for nonparametric testing as appropriate. Statistical tests were two-tailed and a $p$ value of $<0.05$ was considered statistically significant. All the analyses were performed using STATA version 11.2 (StataCorp., College Station, TX, USA).

\section{Ethical considerations}

The study involved human participants and was in accordance with the ethical standards of the institutional and/or national research committee and with the 1964 Helsinki declaration and its later amendments or comparable ethical standards. For this type, informed consent was not required. This study was approved by the Human Research Ethics Committee of Khon Kaen University (HE591128). Patient data were maintained confidentially.

\section{Results}

Twenty-six SSc patients (female to male ratio 3.3:1) who underwent kidney biopsy were included in the study. Most (46.1\%) had the dcSSc subset, followed by SSc overlap SLE $(26.9 \%)$, and lcSSc (15.4\%). Mean age and median duration of disease at the time of kidney biopsy were $53.2 \pm 14.4$ years and 2.4 years (IQR $0.5-7.0$ ), respectively. Nearly $75 \%$ of the patients had pulmonary fibrosis, and around one-third had cardiac involvement at the time of kidney biopsy. The patient characteristics are presented in Table 1 .

RPGN was the commonest renal manifestation indicating that the patient underwent kidney biopsy (53.9\%), followed by nephritis (19.2\%), and AKI with proteinuria, and nephrotic syndrome (11.5\% each) (Table 2). The renal pathology was LN class IV seven cases (26.9\%), LN class V five cases (19.2\%), SRC five cases (19.2\%), and progressive renal disease in scleroderma two cases $(7.7 \%)$ (Figure 1). The nephrotic syndrome was the most common renal presentation among $\mathrm{LN}$ class $\mathrm{V}$ patients, while RPGN was the most common renal feature among LN class IV and SRC patients $(p=0.001)$. At the time of biopsy, dialysis treatment was significantly higher in SRC patients than in other groups $(p<0.001)$. In addition, SRC patients tend to have more frequent cardiac involvement and pulmonary fibrosis and shorter disease duration than the other groups, albeit the differences were not statistically significant.

Figure 2 demonstrates the typical renal pathological findings in SRC. Of the 5 patients who had SRC from renal biopsy 1 diagnosed with $1 \mathrm{cSSc}$ and had clinical features of SRC at the first visit, 3 had normal blood pressures, 1 had nephrotic range proteinuria, and 1 had active urine sediment but none had low complement. Only 3 of those who had LN class IV had active urine sediment; 1 had a low complement level, and 2 had clinical resembling SRC, and 2 of the 5 who were diagnosed as LN class $\mathrm{V}$ had urine sediment (Table 2).

The majority of patients $(87 \%)$ were positive for the anti-topoisomerase antibody. Anti-dsDNA was positive in 1 patient who was diagnosed with SSc overlap SLE and had pathological findings compatible with LN class IV. Anti-Sm was positive in three SSc overlap SLE patients, two were LN class IV, and one had diabetic nephropathy. The clinical comparison by renal pathological findings is presented in Table 2.

\section{Discussion}

Renal involvement in SSc can present with only mild proteinuria or as severe and life-threatening. Kidney biopsy is the gold standard for making a definite diagnosis of renal disease. There are, however, some limitations for performing renal biopsy among SSc patients, including patient safety, patient decision, and facilities at the medical 


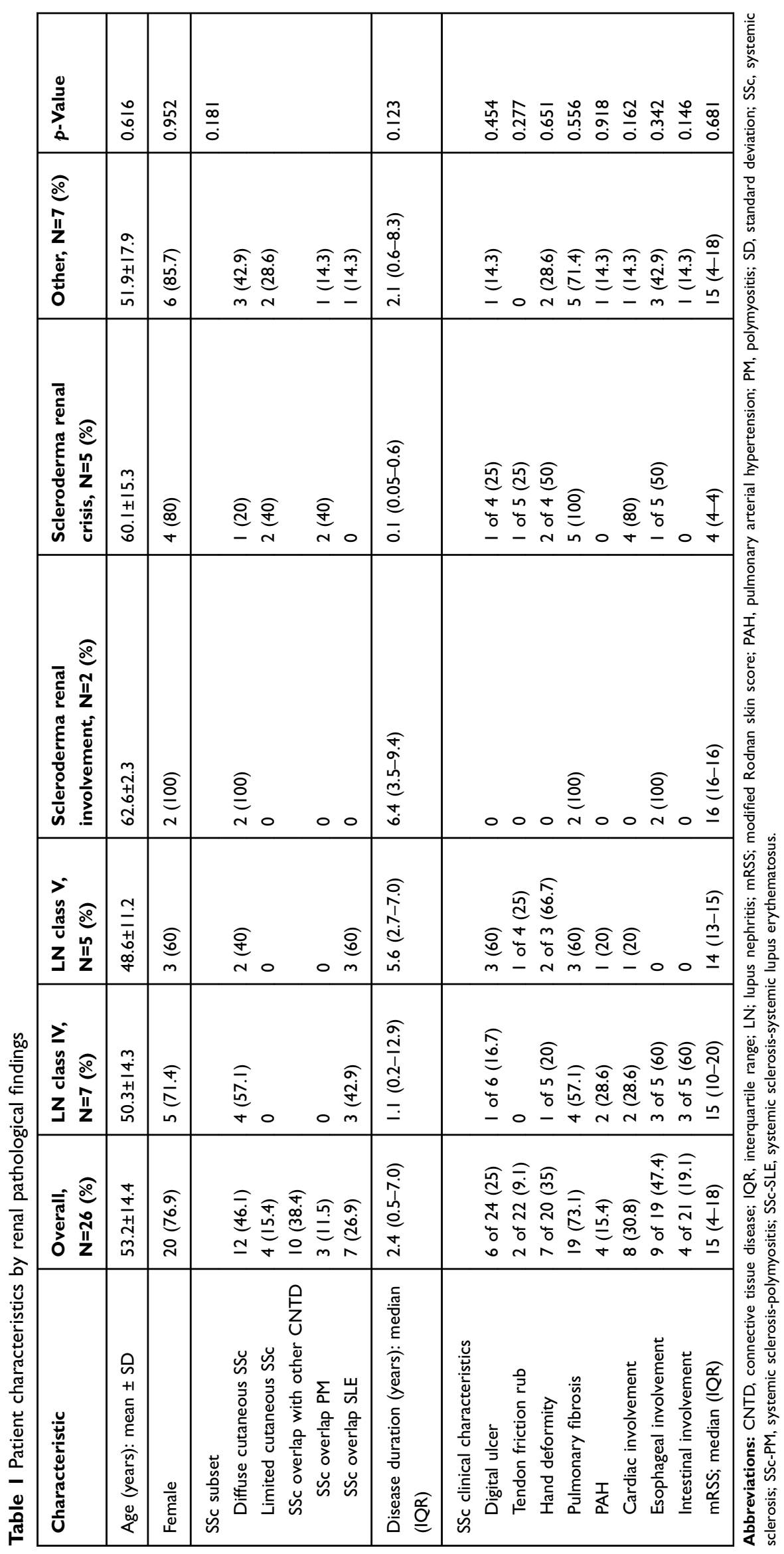




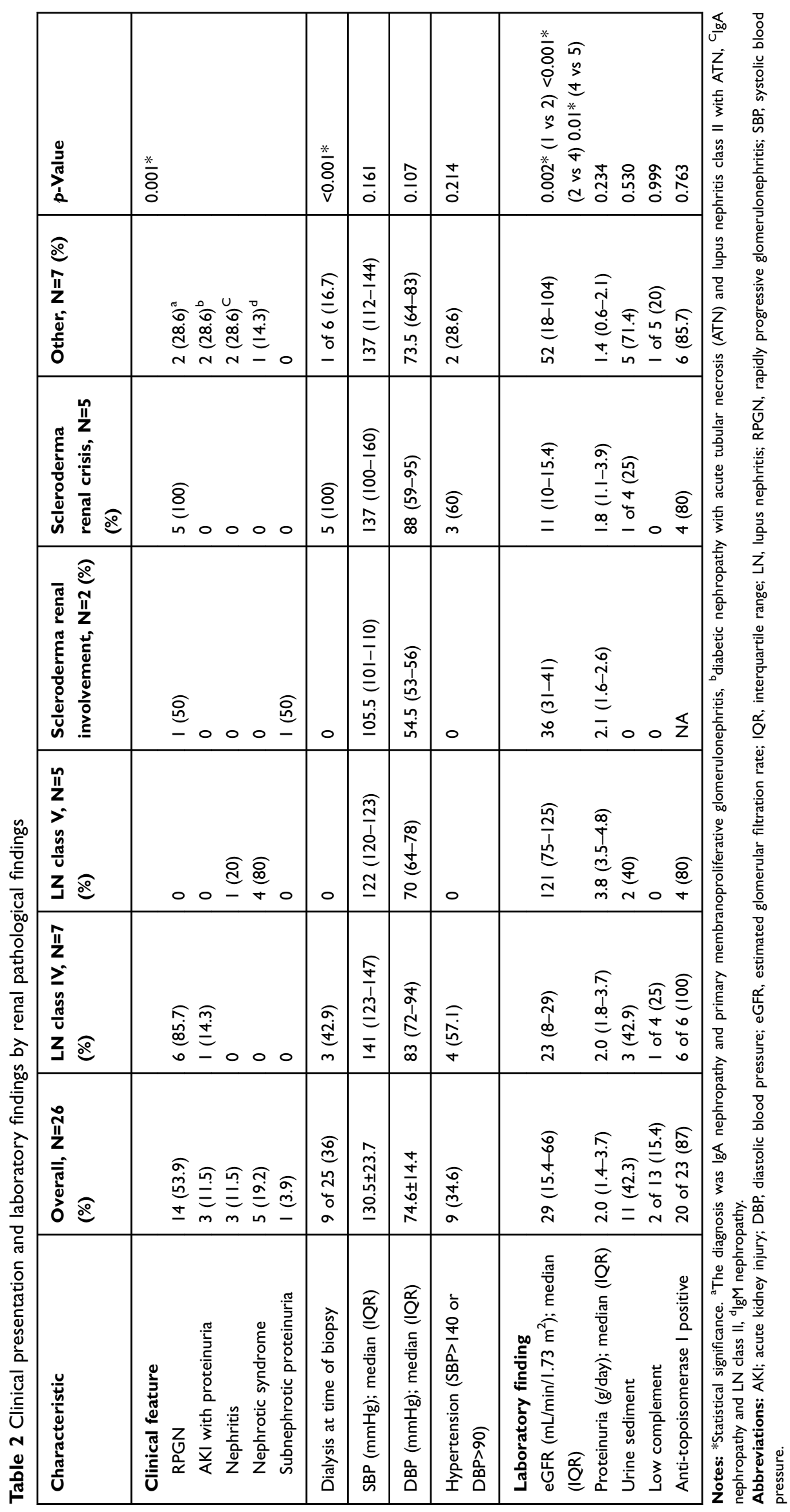




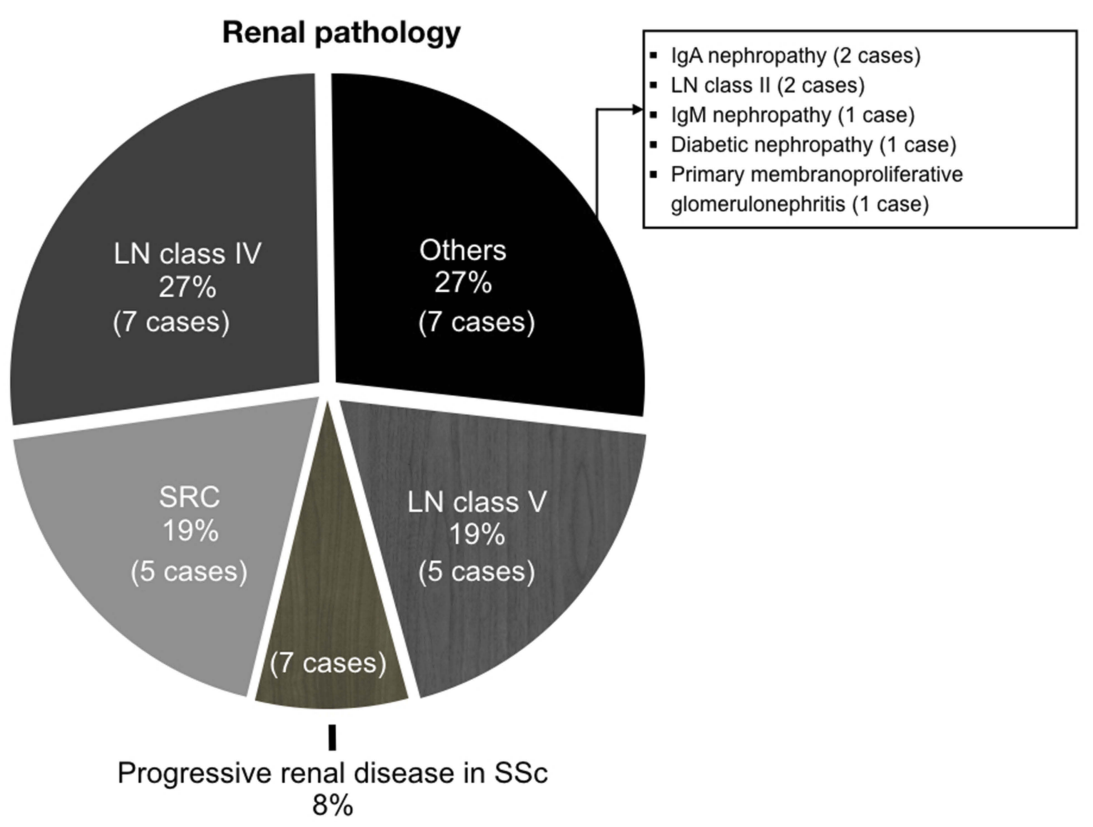

Figure I Renal pathological diagnosis in systemic sclerosis patients.
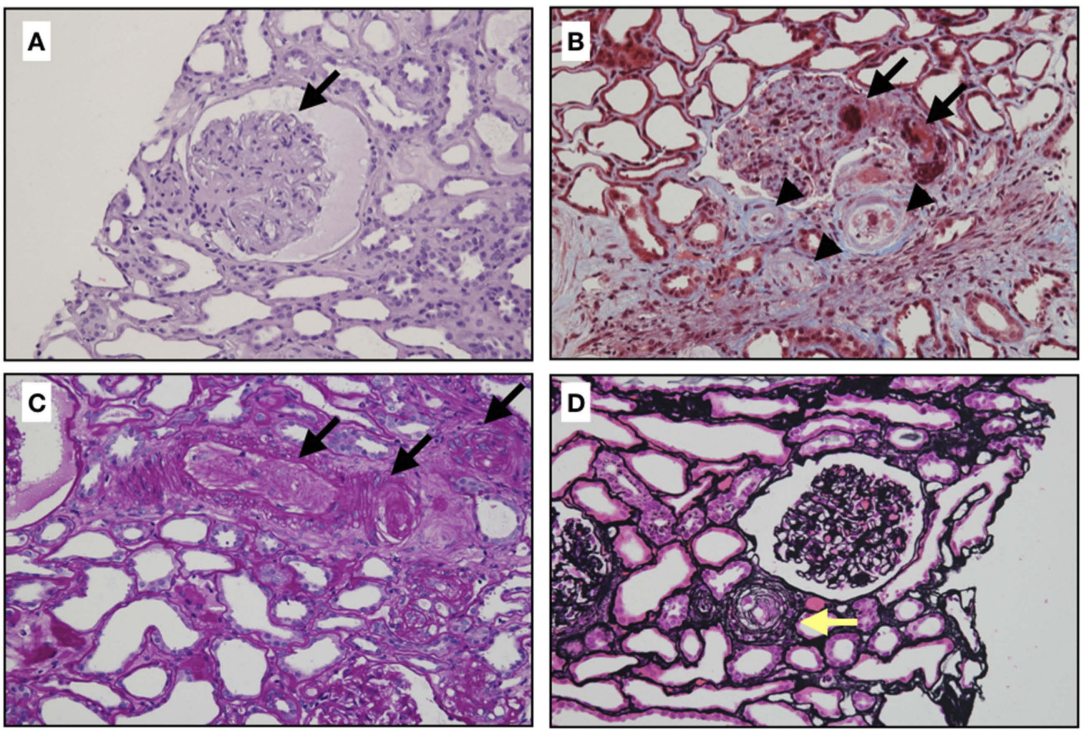

Figure 2 Classic renal pathology in scleroderma renal crisis. (A) The glomerulus demonstrates the global collapse of the glomerular capillary with bloodless appearance, finding similar to ischemic changes. There is no crescent or proliferative lesion (Hematoxylin and eosin stain, $x 400$ ). (B) This figure shows extensive fibrinoid necrosis and fibrin thrombi in afferent arteriole and glomerulus (arrows), and mucoid intimal edema of small arteries (arrow heads) (Masson's trichrome, original magnification x400). (C) Obliteration of arterioles and interlobular arteries (arrows) with thrombi (Periodic acid-Schiff stain, original magnification x400). (D) Intimal concentric lamination (arrow) or onion skin appearance of arteriole (Methenamine silver stain, original magnification $\times 400$ ).

center. These factors might have been the cause of a low rate of kidney biopsy in our study. Nevertheless, this is the first report that reviews the renal pathologic diagnosis and demonstrates the clinicopathological correlation among Thai SSc patients, who have a much greater prevalence of the dcSSc subset than in some Asian and Caucasian SSc patients. $^{10,16,33-36}$
Based on the results, the renal pathology in SSc varies; we found a broad spectrum of involvements including renal involvement in scleroderma, and SSc overlap with SLE and other glomerular diseases include IgA nephropathy, IgM nephropathy, and primary MPGN. These findings indicated that glomerular diseases other than SSc overlap SLE should also be considered in the SSc patient 
with proteinuria with or without AKI. A previous study revealed that chronic tubulointerstitial disease is a common renal pathologic finding in Japanese SSc patients, while glomerular disease was found in a minority. ${ }^{37}$ In contrast to our study, tubulointerstitial disease was not detected, possibly because of differences in the renal manifestation of the patients and indications for kidney biopsy.

$\mathrm{LN}$ is a common renal pathology in our SSc patients $(46.2 \%)$ which reflects that the patients were SSc overlap SLE. The prevalence of SSc-SLE overlap was much higher than prior studies $(6.8 \%$, Alharbi et al and 16\%, Foocharoen et al). ${ }^{17,38}$ Among these patients, 50\% were diagnosed SSc overlap SLE before performing kidney biopsy. These findings suggest that some SSc overlap SLE patients did not have the extra-renal manifestation of SLE, and the diagnosis was enhanced by the kidney biopsy.

Although SRC is the most common renal complication in SSc, only $19 \%$ of our patients had pathology-diagnosed SRC. The explanation might be the low rate of renal biopsy in the patients with clinically suggestive SRC. According to our experience, SRC commonly has a low platelet count, high blood pressure, and/or hemodynamic instability (i.e., from pulmonary edema and cardiac arrhythmias), ${ }^{13}$ so awareness of patient safety is a limitation to perform the procedure. Moreover, SRC is a clinical diagnosis, ${ }^{39,40}$ hence only a minority of patients who had clinically suspected SRC underwent the renal biopsy. Additionally, all of the patients who had pathology consistent with SRC needed dialysis at the time of diagnosis. This finding confirms that SRC is a life-threatening renal complication and causes high morbidity and mortality in $\mathrm{SSc}$; thus, treatment should be prompt for patients with clinically suspected SRC even if there is no pathology proof.

Patients who had SRC had more frequent internal organ involvement - particularly cardiac involvement and pulmonary fibrosis - and shorter disease duration than other groups of patients, albeit the differences were not statistically significant. The result suggests that physicians should monitor for SRC in SSc patients even at an early stage of disease as other internal organ involvements could occur.

Based on our findings of clinicopathological correlation, the presence of nephrotic proteinuria with inactive or bland urine sediment (urine $\mathrm{RBC} \leq 5 / \mathrm{HPF}$ ) and normal GFR indicates non-proliferative glomerulonephritis, which is common in LN class $\mathrm{V}$, whereas the presence of proteinuria with active urine sediment (urine $\mathrm{RBC}>10$ / HPF) and reduction of GFR are commonly associated with proliferative glomerulonephritis, typically LN class IV. SRC typically presents with abruptly elevated serum creatinine, microangiopathy, and hypertension. Urinalysis may show bland or minimal proteinuria $(<1 \mathrm{gm} / \mathrm{d})$ or few microscopic hematuria, ${ }^{41}$ but the nephrotic range of proteinuria is rare. ${ }^{42}$ According to our study, 3 of 5 SRC cases had normal blood pressure, 1 had urine sediment, and 1 had nephrotic range proteinuria ( 2 of whom had LN class IV clinical resembling SRC without features of proliferative LN). In addition, 2 of the 5 cases diagnosed LN class $\mathrm{V}$ had renal clinical features compatible with proliferative LN. The results suggest that uncommon renal clinical features can be present in SSc patients. Besides, the clinical suspicion and histopathological finding might be a discordance in clinical practice. The diagnostic test for evaluation the sensitivity, specificity and predictive values can help to identify the clinical and laboratory predictors of each histopathological finding. Because of low number of each renal involvement particularly SRC in our study, the diagnostic test study is limited by statistical analysis. We do suggest including the large sample to investigate the sensitivity of clinical and laboratory findings for histopathological diagnosis of renal involvement in SSc.

The treatment of renal involvement in SSc depends on the nature of renal disease. SRC is controlled by using angiotensin-converting enzyme inhibitor (ACEI) and supportive treatment, whereas the treatment of choice in $\mathrm{LN}$ is steroid with or without immunosuppressive agents. ${ }^{2,8}$ Therefore, knowing the renal pathology is necessary before determining the nature of renal disease in SSc patients and as a guide for proper treatment. Although the clinical presentation, laboratory data, and serologic investigation can be used for differentiating the renal diseases, ${ }^{15,16,41,43}$ these are insufficient and unreliable evidences for making a definite accurate diagnosis in some situations. Based on our study, we would recommend performing a renal biopsy for all SSc patients with active urinary sediment with or without proteinuria $>1 \mathrm{gm} /$ day so as to exclude other glomerular diseases.

There are some limitations to this study. First, the results are not generalizable as the performance of renal biopsy had a selection bias. Although some patients underwent biopsy with sub-nephrotic range proteinuria or mild symptoms, the majority of renal biopsies were performed in patients with RPGN, nephrotic syndrome, or AKI, which indicated severe renal complications; thus, the rate 
of renal biopsy in our study would be underestimated. Second, some data were missing as is common with retrospective studies. Third, the patients and renal outcomes were not observed, so we cannot provide the association between renal pathology and renal outcomes. Fourth, we did not investigate the association between serology and kidney histopathology because of data limitation. Nevertheless, our study provides information on the clinical features for both renal disease from SSc and renal disease from associated diseases. The results will be helpful when assessing renal disease in SSc patients and determining the most appropriate care in daily practice.

\section{Conclusion}

This study is the first report of renal pathologic findings in Thai SSc patients. RPGN is the most frequent renal manifestation among SSc who underwent kidney biopsy; for whom LN was the most common pathological finding. Nephrotic syndrome is a clinical feature of glomerular diseases other than renal involvement in SSc. Although the clinical and laboratory findings typically predict the glomerular inflammation and pathology, the discordance of clinicopathological association might be recognized in real practice.

\section{Acknowledgments}

The authors thank the Faculty of Medicine and the Scleroderma Research Group, Khon Kaen University, for its support and Mr. Bryan Roderick Hamman for assistance with the English-language presentation under the aegis of the Publication Clinic Khon Kaen University, Thailand.

\section{Disclosure}

The authors declare no conflicts of interest in this work.

\section{References}

1. Ranque B, Mouthon L. Geoepidemiology of systemic sclerosis. Autoimmun Rev. 2010;9(5):A311-A318. doi:10.1016/j.autrev.2009.11. 003

2. Woodworth TG, Suliman YA, Li W, Furst DE, Clements P. Scleroderma renal crisis and renal involvement in systemic sclerosis. Nat Rev Nephrol. 2016;12(11):678-691. doi:10.1038/nrneph.2016.124

3. Traub YM, Shapiro AP, Rodnan GP, et al. Hypertension and renal failure (scleroderma renal crisis) in progressive systemic sclerosis. Review of a 25-year experience with 68 cases. Medicine (Baltimore). 1983;62(6):335-352. doi:10.1097/00005792-198311000-00001

4. Steen VD, Syzd A, Johnson JP, Greenberg A, Medsger TA Jr. Kidney disease other than renal crisis in patients with diffuse scleroderma. $J$ Rheumatol. 2005;32(4):649-655.
5. Trostle DC, Bedetti CD, Steen VD, Al-Sabbagh MR, Zee B, Medsger TA Jr. Renal vascular histology and morphometry in systemic sclerosis. A case-control autopsy study. Arthritis Rheum. 1988;31(3):393400. doi:10.1002/art.1780310311

6. Denton CP, Lapadula G, Mouthon L, Muller-Ladner U. Renal complications and scleroderma renal crisis. Rheumatology (Oxford). 2009;48(Suppl 3):iii32-iii35. doi:10.1093/rheumatology/ken483

7. Wangkaew S, Lertthanaphok S, Puntana S, Noppakun K. Risk factors and outcome of Thai patients with scleroderma renal crisis: a disease duration-matched case control study. Int J Rheum Dis. 2017;20 (10):1562-1571. doi:10.1111/1756-185X.13145

8. Shanmugam VK, Steen VD. Renal disease in scleroderma: an update on evaluation, risk stratification, pathogenesis and management. Curr Opin Rheumatol. 2012;24(6):669-676. doi:10.1097/BOR.0b013e328 $3588 \mathrm{dcf}$

9. Turk M, Pope JE. The frequency of scleroderma renal crisis over time: a metaanalysis. J Rheumatol. 2016;43(7):1350-1355. doi:10. 3899/jrheum. 151353

10. Foocharoen C, Mahakkanukrauh A, Suwannaroj S, Nanagara R. Spontaneous skin regression and predictors of skin regression in Thai scleroderma patients. Clin Rheumatol. 2011;30(9):1235-1240. doi:10.1007/s10067-011-1744-z

11. O'Callaghan CA. Renal manifestations of systemic autoimmune disease: diagnosis and therapy. Best Pract Res Clin Rheumatol. 2004;18 (3):411-427. doi:10.1016/j.berh.2004.03.002

12. Guillevin L, Berezne A, Seror R, et al. Scleroderma renal crisis: a retrospective multicentre study on 91 patients and 427 controls. Rheumatology (Oxford). 2012;51(3):460-467. doi:10.1093/rheumatology/ker271

13. Steen VD. Kidney involvement in systemic sclerosis. Presse Med. 2014;43(10 Pt 2):e305-e314. doi:10.1016/j.lpm.2014.02.031

14. Bose N, Chiesa-Vottero A, Chatterjee S. Scleroderma renal crisis. Semin Arthritis Rheum. 2015;44(6):687-694. doi:10.1016/j.semarthrit. 2014.12.001

15. Denton CP. Renal manifestations of systemic sclerosis - clinical features and outcome assessment. Rheumatology (Oxford). 2008;47 (Suppl 5):v54-v56. doi:10.1093/rheumatology/ken307

16. Panicheewa S, Chitrabamrung S, Verasertniyom O, et al. Diffuse systemic sclerosis and related diseases in Thailand. Clin Rheumatol. 1991;10(2):124-129.

17. Foocharoen C, Netwijitpan S, Mahakkanukrauh A, Suwannaroj S, Nanagara R. Clinical characteristics of scleroderma overlap syndromes: comparisons with pure scleroderma. Int $J$ Rheum Dis. 2016;19(9):913-923. doi:10.1111/1756-185X.12884

18. Foocharoen C, Mahakkanukrauh A, Suwannaroj S, Nanagara R. Prevalence and clinical features of acute kidney injury in Thai systemic sclerosis patients. KKU Med J. 2016;2:29-35.

19. Luciano RL, Moeckel GW. Update on the native kidney biopsy: core curriculum 2019. Am J Kidney Dis. 2019;73(3):404-415. doi:10.1053/j.ajkd.2018.10.011

20. [No authors listed]. Preliminary criteria for the classification of systemic sclerosis (scleroderma). Subcommittee for scleroderma criteria of the American Rheumatism Association Diagnostic and Therapeutic Criteria Committee. Arthritis Rheum. 1980;23(5):581590. doi:10.1002/art.1780230510

21. van Den Hoogen F, Khanna D, Fransen J, et al. 2013 classification criteria for systemic sclerosis: an American College of Rheumatology/European League Against Rheumatism collaborative initiative. Ann Rheum Dis. 2013;72(11):1747-1755. doi:10.1136/ annrheumdis-2013-204424

22. LeRoy EC, Black C, Fleischmajer R, et al. Scleroderma (systemic sclerosis): classification, subsets and pathogenesis. J Rheumatol. 1988;15(2):202-205.

23. Iaccarino L, Gatto M, Bettio S, et al. Overlap connective tissue disease syndromes. Autoimmun Rev. 2013;12(3):363-373. doi:10.10 16/j.autrev.2012.06.004 
24. Pakozdi A, Nihtyanova S, Moinzadeh P, Ong VH, Black CM, Denton CP. Clinical and serological hallmarks of systemic sclerosis overlap syndromes. J Rheumatol. 2011;38(11):2406-2409. doi:10.3899/jrheum. 101248

25. Mukerjee D, St George D, Knight C, et al. Echocardiography and pulmonary function as screening tests for pulmonary arterial hypertension in systemic sclerosis. Rheumatology (Oxford). 2004;43 (4):461-466. doi:10.1093/rheumatology/keh067

26. Ntoumazios SK, Voulgari PV, Potsis K, Koutis E, Tsifetaki N, Assimakopoulos DA. Esophageal involvement in scleroderma: gastroesophageal reflux, the common problem. Semin Arthritis Rheum. 2006;36 (3):173-181. doi:10.1016/j.semarthrit.2006.08.002

27. Savarino E, Furnari M, de Bortoli N, et al. Gastrointestinal involvement in systemic sclerosis. Presse Med. 2014;43(10 Pt 2):e279-e291. doi:10.1016/j.lpm.2014.03.029

28. Group KAW. KDIGO clincial practice guideline for acute kidney injury. Kidney Int Suppl. 2012;17:1-138.

29. Emmett M, Fenves AZ, Schwartz JC. Approach to the patient with kidney disease. In: Skorecki K, Chertow GM, Marsden PA, Taal MW, $\mathrm{Yu}$ ASL, editors. Brenner and Rector's the Kidney. Vol. 1. Philadelphia, PA: Elsevier; 2016:754-779.

30. Weening JJ, D'Agati VD, Schwartz MM, et al. The classification of glomerulonephritis in systemic lupus erythematosus revisited. $\mathrm{J} \mathrm{Am}$ Soc Nephrol. 2004;15(2):241-250. doi:10.1097/01.asn.0000108969. $21691.5 \mathrm{~d}$

31. Fogo AB. Vascular disease. In: Fogo AB, Kashgarian M. editors. Diagnosis Atlas of Renal Pathology. 3rd ed. Philadelphia, PA: Elesvier; 2017:295-364

32. Cannon PJ, Hassar M, Case DB, Casarella WJ, Sommers SC, LeRoy EC. The relationship of hypertension and renal failure in scleroderma (progressive systemic sclerosis) to structural and functional abnormalities of the renal cortical circulation. Medicine (Baltimore). 1974;53 (1):1-46. doi:10.1097/00005792-197401000-00001

33. Allcock RJ, Forrest I, Corris PA, Crook PR, Griffiths ID. A study of the prevalence of systemic sclerosis in northeast England. Rheumatology (Oxford). 2004;43(5):596-602. doi:10.1093/rheumatology/keh124
34. Mayes MD, Lacey JV Jr., Beebe-Dimmer J, et al. Prevalence, incidence, survival, and disease characteristics of systemic sclerosis in a large US population. Arthritis Rheum. 2003;48(8):2246-2255. doi:10. 1002/art. 11073

35. Coral-Alvarado P, Pardo AL, Castano-Rodriguez N, RojasVillarraga A, Anaya JM. Systemic sclerosis: a world wide global analysis. Clin Rheumatol. 2009;28(7):757-765. doi:10.1007/ s10067-009-1144-9

36. Ferri C, Valentini G, Cozzi F, et al. Systemic sclerosis: demographic, clinical, and serologic features and survival in 1,012 Italian patients. Medicine (Baltimore). 2002;81(2):139-153. doi:10.1097/00005792200203000-00004

37. Ichikawa K, Konta T, Sato H, Ueda Y, Yokoyama H. The clinical and pathological characteristics of nephropathies in connective tissue diseases in the Japan Renal Biopsy Registry (J-RBR). Clin Exp Nephrol. 2017;21(6):1024-1029. doi:10.1007/s10157-0171398-5

38. Alharbi S, Ahmad Z, Bookman AA, et al. Epidemiology and survival of systemic sclerosis-systemic lupus erythematosus overlap syndrome. $J$ Rheumatol. 2018;45(10):1406-1410. doi:10.3899/jrh eum. 170953

39. Steen VD, Mayes MD, Merkel PA. Assessment of kidney involvement. Clin Exp Rheumatol. 2003;21(3 Suppl 29):S29-S31.

40. Penn H, Howie AJ, Kingdon EJ, et al. Scleroderma renal crisis: patient characteristics and long-term outcomes. QJM. 2007;100 (8):485-494. doi:10.1093/qjmed/hcm052

41. Ghossein C, Varga J, Fenves AZ. Recent developments in the classification, evaluation, pathophysiology, and management of scleroderma renal crisis. Curr Rheumatol Rep. 2016;18(1):5. doi:10.1007/ s11926-015-0551-y

42. Nepal M, Mainali R, Schworer CM, Difilippo W, Zhang PL, Schultz MF. Nephrotic range proteinuria: rare manifestation of scleroderma renal crisis. Ann Clin Lab Sci. 2008;38(2):163167.

43. Hamaguchi Y. Autoantibody profiles in systemic sclerosis: predictive value for clinical evaluation and prognosis. J Dermatol. 2010;37 (1):42-53. doi:10.1111/j.1346-8138.2009.00762.x
International Journal of General Medicine

\section{Publish your work in this journal}

The International Journal of General Medicine is an international, peer-reviewed open-access journal that focuses on general and internal medicine, pathogenesis, epidemiology, diagnosis, monitoring and treatment protocols. The journal is characterized by the rapid reporting of reviews, original research and clinical studies across all disease areas. The manuscript management system is completely online and includes a very quick and fair peer-review system, which is all easy to use. Visit http://www.dovepress.com/ testimonials.php to read real quotes from published authors. 